\title{
Medical management of extra-ocular muscle cysticercosis- a clinical study
}

\author{
Mishra A. ${ }^{1}$, Singh M. ${ }^{2}$, Agarwal A. ${ }^{3}$, Bajpai V. ${ }^{4}$, Mishra V. ${ }^{5}$ \\ ${ }^{1}$ Dr. Amrita Mishra, Associate Professor, ${ }^{2}$ Dr. Manmeet Singh, Junior Resident ${ }^{3}$ Dr. Akhil Agarwal, Consultant; All \\ authors ${ }^{1,2,3}$ are affiliated with Department of Ophthalmology, Shri Ram Murti Smarak Institute of Medical Sciences, \\ Bareilly, Uttar Pradesh, ${ }^{4}$ Dr. Vijeta Bajpai, Consultant, Anaesthesia, SGPGI, Lucknow, UP, India, ${ }^{5}$ Dr. Vashishth Mishra, \\ Associate Professor, Microbiology, Government Medical College, Badaun, Uttar Pradesh.
}

Corresponding Author: Dr. Vashishth Mishra, Associate Professor, Microbiology, Government Medical College, Badaun, Uttar Pradesh, India. E-Mail: amrita.gsvm@gmail.com, drbravesingh@gmail.com

\begin{abstract}
Introduction: To study clinical diagnosis, results of investigations and role of medical management and their outcome in extra-ocular cysticercosis. Our study also emphasizes on the role of topical cyclosporine eye drops in the management of treatment related severe inflammatory response in extra-ocular cysticercosis. Methods: A total of 10 patients with extraocular cysticercosis were recruited for the study from our OPD, blood investigations, ultra-sonography for both eyes and whole abdomen were done. Computed tomography (NCCT) were done to rule out neurocysticercosis and orbital cysticercosis. Results: The commonest clinical presentation was cyst in the medial rectus muscle with it being the most common presenting manifestation. Conclusion: Extra-ocular cysticercosis can be managed with medical treatment. Oral Albendazole, topical and systemic steroids were given as a part of medical treatment, topical cyclosporin was added to the patients with more severe inflammatory response due to dying cysticercus.
\end{abstract}

Key words: Extra-ocular cysticercosis, Medical management, Cyclosporine

\section{Introduction}

Cysticercosis is a serious problem in developing countries of Latin America, Asia and Africa, especially in areas of poverty and poor hygiene. Taenia solium is a member of Phylum Platyhelminthes, class Cestoda Order Cyclophyllidea and family Taeniidae [1].

In our study, we studied the patients with extra-ocular muscle cysticercosis, its presenting symptoms, most commonly involving extra-ocular muscles after ruling out any possibility of intra-ocular cysticercosis. All the patients were treated with medical therapy. The study also highlights the role of topical cyclosporine in the management of inflammatory response occurring due to dying cysticercus.

Medical management of extra-ocular muscle cysticercosis is a good alternative to surgical management as it prevents surgical damage to the muscle involved, post-operative discomfort and long term post-operative medication.

\footnotetext{
Manuscript received: $11^{\text {th }}$ October 2018

Reviewed: $19^{\text {th }}$ October 20178

Author Corrected; $24^{\text {th }}$ October 20178

Accepted for Publication: $29^{\text {th }}$ October 2018
}

Ocular Cysticercosis- Clinical Presentation- Ocular involvement is usually unilateral but bilateral involvement may occur in cases of disseminated cysticercosis [1].

Left eye is more commonly involved in comparison to the right, possibly because larva may be preferentially routed to the left internal carotid artery which directly originates from the aorta. Parasite reaches the posterior segment through the posterior ciliary artery. Intraocular cysticercosis can involve either the anterior or the posterior segment. While the anterior segment cysticercosis is rarely seen.

Intra-vitreal cysts- Various modalities have been described in the surgical management of intra-vitreal cysticercosis such as diathermy, photocoagulation and cryo-therapy. Surgical removal of the cyst can be through either the trans-retinal or trans-scleral route.

Lid Cysticercosis- Involvement of the eyelids present as a subcutaneous, painless, mobile mass with varying degrees of mechanical ptosis [2]. 


\section{Original Research Article}

\section{Subconjuctival cysticercosis- Conjunctival} involvement is usually in the form of a painless or painful yellowish, nodular subconjunctival mass with surrounding conjunctival congestion. Rarely subconjunctival abscess or granuloma may occur.

Extraocular myo-cysticercosis- Cysticercosis of extraocular muscle usually presents as recurrent pain, redness, proptosis, ocular motility restriction, diplopia and ptosis. One or more extra-ocular muscles may be simultaneously involved.

Optic Nerve- Optic nerve compression by the cyst may cause decreased vision, disc oedema and painful ocular motility [3].

Lacrimal Gland- Lacrimal gland cysticercus may cause a chronic dacryo-adenitis and enlargement of the gland. Lacrimal canalicular obstruction due to adnexal cysticercus has also been reported [4].

Subretinal Cysticercosis- Patients with ocular cysticercosis may be asymptomatic or suffer mild to severe vision loss. Patients presented with painless vision loss secondary tpo a parasitic infection may be presumed due to sub-retinal cysticerosis [5].

Posterior Segment Cysticercosis- In the posterior segment of the eye, vitreous cysts are more common than retinal or subretinal cysts and the infero-temporal subretinal cyst is most frequently encountered.

The macular region being the thinnest and most vascularised, the larvae lodges itself in the subretinal space from where it perforates and enters into the vitreous cavity [6]. In this process, the parasite can cause a retinal detachment, macular hole or incite an inflammatory response. As the cyst develops, it causes atrophic changes of the overlying retinal pigment epithelium. Sometimes, it may cause exudative retinal detachment and focal chorioretinitis. The central retinal artery is the most likely route for cysticercosis involving the optic nerve head.

A dying cysticercosis cyst can incite a severe inflammatory response, due to the leakage of the toxin from the micro- perforations present in the cyst wall. Inflammatory reaction can be present even the living parasite and more so with vitreous cysts than subretinal cysts. Complications of intraocular cysticercosis include severe inflammation (vitreous exudates, organised membranes in vitreous), retinal detachment, complicated cataract, hypotony and phthisis. It is seen that the involvement of the posterior segment is common [7].

\section{Materials and Methods}

Type of Study- It was a prospective study which comprised of 10 patients with extra-ocular cysticercosis who visited the Out-Patient Department of Ophthalmology.

Place of Study- It was conducted in the Department of Ophthalmology at Shri Ram Murti Smarak Institute of Medical Sciences, Bareilly, Uttar Pradesh

Sample Collection and Duration of Study - It was randomised clinical study on ten patients who presented to us in the Ophthalmology OPD from January 2017 to June 2018 who were included in this study, having had cysticercosis involving the extra-ocular muscles only. Ethical committee clearance was taken. Informed consent was taken from all the participants.

\section{Inclusion Criteria}

$>$ Patients having cysticercosis involving the extraocular muscle only.

\section{Exclusion Criteria}

$>$ Patients with neurocysticercosis

$>$ Patients with intraocular cysticercosis

$>$ Patients with systemic cysticercosis

$>$ Patients with conjunctival cysticercosis

Sampling Methods- During the first visit, information recorded for each case included: age, sex, occupation, detailed history, regarding symptoms and duration of onset, course of the disease, eye involvement, visual status after the onset of symptoms and at presentation, previous investigations and treatment. Detailed ophthalmic examination was performed on all the patients.

Torch light examination and slit lamp bio-microscopy was done for the anterior segment evaluation and indirect ophthalmoscopy for the posterior segment. General physical and neurological examination was also conducted.

Depending on the location of the cyst, the relevant clinical tests performed were: diplopia charting, ptosis work-up and Hertel's exophthalmometry.

All patients underwent ultrasonography USG A and B scan (eye and orbit) and computed tomography (CT) with $2 \mathrm{~mm}$ sections, head and orbit; axial and coronal cuts. Typically, A scan ultrasonography shows high amplitude spikes corresponding to the cyst wall and 


\section{Original Research Article}

scolex and B scan ultrasonography shows hanging drop sign i.e. echoes corresponding to the cyst wall with the scolex attached to the inner wall. CT scan shows a non- enhanced circular area of low attenuation with a tiny area of increased attenuation within the lesion which is pathognomic of scolex.

\section{Results}

Out of 10, nine patients had resolved extra-ocular cysticercosis with this medical management given for 4 weeks with the use of topical cyclosporine, the inflammatory response was better controlled when given in addition to the routine medical regimen. For one patient the treatment was prolonged for 6 weeks, which was later resolved. It was found that the male preponderance was more in comparison to females, with complaint of redness being the most common presenting symptom followed by lid swelling and pain involving levator palpebrae superioris and medial rectus being the most commonly involved muscles.

Table-1: Sex Distribution among patients

\begin{tabular}{|c|c|}
\hline Total No. of patients & $\mathbf{1 0}$ \\
\hline Male & 7 \\
\hline Female & 3 \\
\hline
\end{tabular}

Male patients were more commonly involved in extra-ocular cysticercosis

Table-2: Age Distribution among the patients

\begin{tabular}{|c|c|}
\hline Age & No. of patients \\
\hline $0-10$ & 5 \\
\hline $11-20$ & 3 \\
\hline $21-30$ & 2 \\
\hline
\end{tabular}

Younger individuals less $<10$ yrs of age were more commonly affected, poor awareness towards hygiene in this age group can be a related reason to this.

Table-3: Comparison on the basis of symptoms

\begin{tabular}{|c|c|}
\hline Symptoms & No. of patients \\
\hline Lid swelling & 3 \\
\hline Pain & 3 \\
\hline Redness & 5 \\
\hline Diplopia & 2 \\
\hline
\end{tabular}

Red eye was the most common symptom amongst majority of patients.

Table-4: Incidence on the basis of muscle involved

\begin{tabular}{|c|c|}
\hline Extra-ocular muscle involved & No. of patients \\
\hline LPS & 3 \\
\hline Medial Rectus & 3 \\
\hline Lateral Rectus & 2 \\
\hline Superior Rectus & 1 \\
\hline Inferior Rectus & 1 \\
\hline
\end{tabular}

Levator Palpebrae Superioris and Medial Rectus muscle were more commonly involved extra-ocular muscle.

Treatment regimen given in this study was oral Albendazole in dosage of 15-20 mg/kg/day, oral Prednisolone acetate (1$1.5 / \mathrm{kg}$ body wt.) in addition to which topical corticosteroids and topical cyclosporine as added to control the inflammatory response thereof. 


\section{Discussion}

Prevalence- Factors facilitating the spread of T. solium infection include inadequate sanitation, breeding pigs in unsanitary conditions, and eating uncooked pork. Risk factors include a family history of parasitic infestation, history of travel to an endemic area, or household visitors from an endemic area. Cysticercosis affects an estimated 50 million people worldwide. Ocular cysticercosis is endemic in tropical areas, such as subSaharan Africa, India, and East Asia. In India, 78\% of the cases with ocular cysticercosis have been reported from states of Andhra Pradesh and Pondicherry [1].

Cysticercosis may cause significant visual loss, especially if the cyst is located intraocularly or is compressing the optic nerve. There is no specific sex predilection. People of any age may be affected, although orbital cysticercosis is more commonly reported in younger age groups. Other endemic areas include Mexico, Latin America, China, Indonesia and Eastern Europe [8].

Morphology, Biology and Life Cycle- Tapeworms can cause two different human diseases, taeniasis and cysticercosis. Taeniasis is an intestinal infection caused by the adult T. solium and Taenia saginata of Phylum Platyhelminthes, class Cestoda Order Cyclophyllidea and family Taeniidae [9].

Cysticercosis, the most common ocular infestation in humans, is caused by encystment of the larvae (cysticercus cellulosae) of the tapeworm T. solium, in which humans are the intermediate hosts in the life cycle [10].

The incubation period may vary from months to years. The ocular manifestations can be devastating as the cysticercus increases in size, leading to blindness in 3-5 years. Death of the parasite causes marked release of toxic products, leading to a profound inflammatory reaction and destruction of the eye. Appropriate sanitation and personal hygiene are important in control of fecal contamination of water and food. Humans become infected when they ingest raw or undercooked pork that contain viable cysticerci.

The cysticercus larvae is semi-transparent, opalescent white and elongate, oval in shape and may reach a length of 0.6 to $1.8 \mathrm{~cm}$. Human cysticercosis occurs when T. solium eggs are ingested via faecal-oral transmission from a tapeworm infected host. The human then becomes an accidental intermediate host. These oncospheres (primary larvae) penetrate the intestinal mucosa and enter the circulatory system. Haematogenous spread to neural, muscular, and ocular tissues occurs. Within these tissues, the oncospheres develop into secondary larvae (cysticerci). The host inflammatory response to cystercerci depends on the parasite's ability to evade host immunity [11].

Ocular involvement is usually unilateral, but bilateral involvement may occur in cases of disseminated cysticercosis [12]. Left eye is more commonly involved in comparison to the right, possibly because larva may be preferentially routed to the left internal carotid artery which directly originates from the aorta; however, this has not been substantially proven [13].

The medial side of the eye has been more commonly involved than the lateral side on account of the anatomic course of the ophthalmic artery, which after giving off the lacrimal branch runs on the medial side of the orbit before diving into the terminal branches. Infestation of the ocular adenexa is probably through the anterior ciliary artery. Parasite reaches the posterior segment through the posterior ciliary artery [14].

Extraocular myocysticercosis: cysticercosis of extraocular muscle usually presents as recurrent pain, redness, proptosis, ocular motility restriction, diplopia, and ptosis. One or more extraocular muscles may be simultaneously involved although a propensity for involvement of the superior muscle complex and the lateral rectus muscles has been reported [15].

T. solium releases three to six proglottids/day, bearing 30,000 to 70,000 eggs per proglottid into the intestine. The adult worm may live in the small intestine for as long as 25 years without symptoms (taeniasis) and pass gravid proglottids intermittently with the faeces [16].

The cysticercus larvae is semitransparent, opalescent white, and elongate oval in shape and may reach a length of 0.6 to $1.8 \mathrm{~cm} .4$ Human cysticercosis occurs when $T$. solium eggs are ingested via faecal-oral transmission from a tapeworm infected host. The human then becomes an accidental intermediate host. These oncospheres (primary larvae) penetrate the intestinal mucosa and enter the circulatory system. The cysts are usually multiple and may be deposited in any tissue, the eye, orbit, and nervous system being most frequently affected. The embryo forms a globular translucent cyst which causes a foreign body reaction, and if it is ruptured a suppurative inflammation occurs that may destroy the eye. 
Diagnosis- The diagnosis of myocysticercosis is based on clinical, serologic, and radiological findings. The clinical findings may occasionally be non-specific and hence, non diagnostic. Serological tests used for the specific diagnosis of cysticercosis are indirect hemagglutination, indirect immuno-fluorescence, and immuno-electrophoresis such as ELISA specific serology. The serology in myocysticercosis may show false positive reports. Thus, imaging studies are the most helpful in establishing the diagnosis of cysticercosis. High resolution Ultrasonography (USG), computed tomography (NCCT) and Magnetic Resonance Imaging (MRI) help in detection of the orbital cyst.

Diagnosis of infection with adult T. solium is made by stool examination and finding the eggs of proglottids of the worm. Though stool examination for the adult worm may be performed in cases of suspected myocysticercosis infections, it is not essential that all patients with myocysticercosis have the adult worm in their intestines except in those cases, which are acquired by auto-infection. B-scan ocular ultrasonography reveals a well-defined cystic lesion with clear contents and a hyperechoic area suggestive of a scolex [17].

Typically, A-scan USG shows high amplitude spikes corresponding to the cyst wall and scolex. The scolex shows a high amplitude spike due to presence of calcareous corpuscles [18].

Ocular ultrasonography is a useful tool for diagnosis and monitoring of the cyst during treatment. CT scanning of the orbits is a reliable technique to help establish a diagnosis of ocular cysticercosis. The characteristic feature is a hypodense mass with a central hyperdensity suggestive of the scolex. Usually, a solitary cyst with wall enhancement is observed. Adjacent soft-tissue inflammation may be present. The scolex may not be visible if the cyst is dead or ruptured and has surrounding inflammation. Concurrent neurocysticercosis may be present and should be excluded. MRI reveals a hypointense cystic lesion and hyperintense scolex within the extraocular muscle.

Treatment- Albendazole is the larvicidal drug used in the treatment of cysticercosis in human. Once the diagnosis of orbital cysticerocosis is confirmed, it is of utmost importance to rule out intra-ocular and central nervous system involvement. Albendazole is a well tolerated broad spectrum cysticidal drug and destroys approximately $85 \%$ of cysts with a single course. Dying cysticercus releases its toxin and incites severe inflammatory reaction leading to vitritis and may lead to

\section{Original Research Article}

blindness. Hence it is mandatory to check for intraocular involvement of cysticercus cyst. Cure rates range from 60 to $85 \%$ in the usual dosing with most series showing albendazole $70-90 \%$ yielding slightly higher cure rates. Albendazole is converted to its active metabolite, albendazole sulphoxide, in the liver. It is usually given at $15 \mathrm{mg} / \mathrm{kg} /$ day with a maximum of 400 $\mathrm{mg} / \mathrm{bid}$ with repeated dosings as clinically warranted. Absorption of albendazole is increased with fatty foods. Treatment may increase inflammation as the cysts involutes, leading to worsening clinical states. Thus, concomitant administration of corticosteroids is recommended to avert an inflammatory response that usually occurs 2-5 days after initiation of therapy. Eye drop cyclosporine in the patient at the time of inflammatory reaction because of the dying cysticercus was also used.

Orbital cysts are best treated conservatively with a 4 week regimen of oral albendazole $(15 \mathrm{mg} / \mathrm{kg} /$ day $)$ in conjunction with oral steroids $1.5 \mathrm{mg} / \mathrm{kg} / \mathrm{day}$ in a tapering dose over a 1 month period. In our study, 10 patients with extra-ocular muscles with cysticercosis were included, majority of which were below 10 years and the most common symptoms was redness followed by pain and lid swelling. To patients were also having diplopia and extra-ocular muscle movement restriction.

Steinmetz et al.1989 suggested that anti-helminthic drugs like albendazole or praziquantel reduce the number of cysts and the frequency of seizures in neurocysticercosis. [19] However early excision of intra-ocular cysticercosis is the treatment of choice as been quoted by Gemolotto et al in 1955 [20].

Natarajan et al.1999 quoted that if there is co-infection with intra-ocular and intra-cranial cysticercus, the complete intra-ocular cyst must be removed completely by surgery by first, followed by cysticidal drugs and corticosteroids. Anti-helminthic therapy is contraindicated in ocular cysticercosis because lysis and degeneration of intra-ocular cyst may induce intraocular inflammatory response and result in visual loss [21].

The vitreous hemorrhage, a well-known complication of surgery during cyst excision was quoted by Sharma et al.2003 [22].

Cysticercosis can be prevented through practicing good hygiene measures, such as washing hands frequently, washing raw vegetables and fruits well before consumption to prevent faecal-oral transmission and 


\section{Original Research Article}

avoiding consumption of raw or undercooked pork and other meat [23].

\section{Conclusion}

Extra-ocular cysticercosis can be managed with medical treatment provided. Dilated fundus with B/E USG Bscan is mandatory to rule out any intra-ocular cysticercosis to avoid any vision threatening complication of medical management.

Depending upon the response, medical management can be given for 4-6 weeks in addition to topical cyclosporine being added to control the inflammatory response of the dying cysticercus in few patients. Medical management also avoids unnecessary surgical damage to the extra-ocular muscle, severe reaction due to cyst rupture at the time of surgery, long postoperative discomfort and post surgical topical medication.

In our study, topical cyclosporin had given wonderful anti-inflammatory response when added to topical steroids. Thus, medical management of extra-ocular cysticercosis can be a better option to surgical intervention.

\section{Medical management of Extra-ocular Cysticercosis- A preferable alternate}

Role of medical management plays a due importance in curing the patients of extra-ocular cysticercosis and the other side of the coin is the use of Cyclosporine, which can be a better option to control inflammatory reaction giving an upper hand over the prolonged use of steroids.

Acknowledgements- We would like to thank Shri Ram Murti Smarak Trust, Bareilly, Uttar Pradesh, India

Funding: Nil, Conflict of interest: Nil

Permission from IRB: Yes

\section{References}

1. Reddy PS, Satyendran OM. Ocular Cysticercosis. Am J Ophthalmol. 1964 Apr;57:664-6.

2.Topilow HW, Yimoyines DJ, Freeman HM, et al. Bilateral multifocal intraocular cysticercosis. Ophthalmology Nov 1981;88(11) :1166-72.

3. Bawa YS, Wahi PL. cysticercosis cellulosae of the optic disc with generalized cysticercosis. $\mathrm{Br} \quad \mathrm{J}$ Ophthalmol. 1962 Dec;46(12):753-5.
4. Pandey PK, Chaudhuri Z, Sharma P, Bhomaj S. Extraocular muscle cysticercosis: a clinical masquerade. J Pediatr Ophthalmol Strabismus. 2000 Sep-Oct;37 (5):273-8.

5. Lombardo J. Subretinal cysticercosis. Optom Vis Sci. 2001 Apr;78(4):188-94.

6. Sharma T, Sinha S, Shah N, et al. Intraocular cysticercosis: clinical characteristics and visual outcome after vitreoretinal surgery. Ophthalmology. 2003 May;110(5):996-1004.

7. Rath S, Honavar SG, Naik M, et al. Orbital cysticercosis: clinical manifestations, diagnosis, management, and outcome. Ophthalmology. 2010 Mar;117(3):600-5, 605.

8. Pushker N, Bajaj MS, Chandra M, Neena. Ocular and orbital cysticercosis. Acta Ophthalmol Scand. 2001 Aug;79(4):408-13.

9. Miller NR, Newman NJ (eds). Walsh and Hoyt's Clinical Neuro-Ophthalmology. Baltimore: Williams and Wilkins, 1997; 4439-44

10. Markell EK, John DT, Krotoski WA. Medical Parasitology Eighth Edition. Pennsylvania: Saunders, 1999.

11. Davis, LE. "Neurocysticercosis" Emerging Neurological Infections edited by Poer, $\mathrm{C}$ and Johnson RT. Taylor \& Francis Group, 2005.261-287.

12. Pushker N, Mehta M, Meel R, Bajaj MS. Disseminated cysticercosis with multiple bilateral orbita 1 cysts. Ophthalmic Plast Reconstr Surg. 2009 NovDec;25(6):499-501.

13. Das S. Anterior orbital cysticercosis: A case presentation. Kerala J Ophthalmol 2017;29:230-3.

14. Genta RM, Connor DH. Infectious and parasitic diseases. In: Rubin E, Farber J, editors. Pathology. Philadelphia: Lippincott- Raven; 1999. p. 356-479. 10.

15. Sundaram PM, Jayakumar N, Noronha V. Extraocular muscle cysticercosis - a clinical challenge to the ophthalmologists. Orbit. 2004 Dec;23 (4):255-62.

16. Odel JG, Moazami G. Diseases caused by helminths. In: Miller NR, Newman NJ (eds). Walsh and Hoyt's Clinical Neuro-Ophthalmology. Baltimore: Williams and Wilkins, 1997; 4439-44 


\section{Original Research Article}

17. Honavar SG, Sekhar CG. Ultrasonological characteristics of extraocular cysticercosis. Orbit 1998; 17(4):271-84.

18. Rahalkar MD, Shetty DD, Kelkar AB, et al. The many faces of cysticercosis. Clin Radiol. 2000 Sep;55(9):668-74.

19. Steinmetz RL, Masket S, Sidikaro Y. The successful removal of subretinal cysticercus by pars plana Vitrectomy. Retina. 1989;9:276-280.

20. Gemolotto G.A contribution to surgical treatment of intraocular cysticercosis. Arch Ophthalmol 1955;59:365-368.
21. Natarajan S, Malpani A, Kumar Nirmalan P, Dutta B. Management of intraocular cysticercosis. Graefes Arch Clin Exp Ophthalmol. 1999; 237:812-814.

22. Sharma T, Sinha S, Shah N, Gopal L, Shanmugam MP, Bhende P, Bhende M, Shetty NS, Agrawal R, Deshpande D, Biswas J, Sukumar B. Intraocular cysticercosis: clinical characteristics and visual outcome after vitreoretinal surgery. Ophthalmology. 2003;110 (5):996-1004.

23. Sonam Angmo Bodh, Saurabh Kamal, Sushil Kumar Orbital Cysticercosis. Del J Ophthalmol 2012; 23 (2): 99-103.

\section{How to cite this article?}

Mishra A., Singh M., Agarwal A., Bajpai V., Mishra V. Medical management of extra-ocular muscle cysticercosis- a clinical study. Trop J Ophthalmol Otolaryngol.2018;3(4):85-91.doi: 10.17511/jooo.2018.i04.05 